\title{
Accuracy of disorder predictors results - comparison on DisProt DB data
}

\author{
Nenad Mitić \\ Faculty of Mathematics \\ University of Belgrade \\ Belgrade, Serbia \\ nenad@matf.bg.ac.rs \\ Miloš Beljanski \\ Insistute for General and Physical \\ Chemistry \\ University of Belgrade \\ Belgrade, Serbia \\ mbel@matf.bg.ac.rs
}

\author{
Gordana Pavlović-Lažetić \\ Faculty of Mathematics \\ University of Belgrade \\ Belgrade, Serbia \\ gordana@matf.bg.ac.rs
}

\author{
Mirjana Maljković \\ Faculty of Mathematics \\ University of Belgrade \\ Belgrade, Serbia \\ mirjana@matf.bg.ac.rs
}

\author{
Saša Malkov \\ Faculty of Mathematics \\ University of Belgrade \\ Belgrade, Serbia \\ smalkov@matf.bg.ac.rs
}

\author{
Ana Jelović \\ Faculty of Transport and Traffic \\ Engineering \\ University of Belgrade \\ Belgrade, Serbia \\ a.jelovic@sf.bg.ac.rs
}

\begin{abstract}
Some results of accuracy and inconsistencies of disordered predictors prediction compared on proteins regions from DisProt database are presented. Results are compared for more than 20 predictors applied on different versions of DisProt database.
\end{abstract}

\section{Keywords - disorder predictors, accuracy, DisProt database}

\section{Introduction}

It is well known that Intrinsically Disordered Proteins (IDPs) play significant role in key biological processes including signaling, recognition, regulation and cell cycle control [1]. Consequently, they are associated with various human pathologies, such as cancer, diabetes, cardiovascular and neurodegenerative diseases [2] and therefore are in the focus of many researches.

The process of experimentally determining disordered regions in proteins is slow and expensive, resulting in relatively small number of experimentally confirmed disordered regions. Information about such region were collected from the literature (manually curated) in DisProt database of proteins with experimentally detected disorder regions $[3,4,5]$. Number of collected regions vary from 152 proteins with 176 regions in DisProt 1.0 (released in 2003 year) to 1713 proteins with 7902 regions in DisProt V8.01. (released in 2019 year). The number of proteins and regions are even smaller because database include information about ambiguous and obsolete regions. Because importance of IDPs and their roles in many processes, as a consequence of slow and inefficient process of experimentally detecting, large number of computer programs - disorder predictors - has been developed for prediction of disordered regions in proteins.

As the reasons and mechanism of appearing disordered regions in proteins are not theoretically formally described, different methods and bases are used for prediction algorithms in disorder predictors (for example, see [6]). Some predictors behave better in one class of proteins and opposite. But researchers do not have any guarantee which predictors acts better and more accurate on completely new and previously unknown (unclassified) protein. In some cases, applying two or more predictors can lead to contradictory and confusing results.

The aim of this work was to analyze predictors results over experimentally verified disorder regions. This was motivation to apply predictors on proteins collected in DisProt database, and compare obtained results with expected i.e. experimentally confirmed as potentially "stable point".

\section{Matherial and Methods}

Material used in research includes all proteins from DisProt version 1.0 to DisProt Version 8.01. There are a lot of changes between DisProt consecutive versions. The changes include adding and removing proteins that were declared as disordered, obsolete regions, different AA contents of disorder regions and change in fractional differences, change in protein sequence, removing information about ordered regions which exist up to version 6.02, etc. Nevertheless, because DisProt is the one and only available collection of experimentally detected disordered regions it is used as benchmark.

Analysis is done using over 20 most commonly used disorder predictors divided into two group based on the method access possibilities. The first group includes predictors available for download and used in local environment as standalone versions, while the second one includes predictors that can be used over corresponding web sites.

The analysis includes a comparison of predicted and experimentally found disordered regions, as well as different predictors aspects including precision and accuracy at the points on the timeline associated with the detection of the regions and the construction of the predictors.

In addition to use as criteria direct hit (match, intersection) with the positions of the experimentally confirmed regions, other different criteria was used like approximate guessing, i.e. did the predictor predict the position in the environment or nearby the positions of regions in DisProt, method used to identify disordered regions, percentage of matched regions known at the time of predictors were announced and percentage of matched regions known after predictors were announced.

\section{Results}

Analysis produced very different results. Some predictors had very high level of correct predictions, but, as expected, there is no predictor that behaves as "the best" in all cases. Moreover, in some cases when consensus of some predictors was taken, these results did not match positions of 
experimentally detected regions. Some cause of the lower quality of the results of some predictors are possible consequences of change between consecutive DisProt version that was used as benchmark. This is especially evident for difference between percentage of matched regions known before and after predictor announced. Obtained results suggest that for some predictors additional training which takes latest versions of DisProt DB can be useful.

\section{REFERENCES}

[1] V.N. Uversky: "Introduction to Intrinsically Disordered Proteins (IDPs)", Chem Rev. 2014;114(13):6557-60

[2] V.N. Uversky, C.J. Oldfield, and A.K. Dunker: "Intrinsically Disordered Proteins in Human Diseases: Introducing the D2 Concept", Annual Review of Biophysics (2008), Vol. 37:215-246.
[3] S. Vucetic et al. "DisProt: a database of protein disorder", Bioinformatics. 2005 Jan 1;21(1):137-40. Epub 2004 Aug 13.

[4] A. Hatos et al. "DisProt: intrinsic protein disorder annotation in 2020", Nucleic Acids Research, Volume 48, Issue D1, 08 January 2020, Pages D269-D276.

[5] D. Piovesan et al. "DisProt 7.0: a major update of the database of disordered proteins", Nucleic Acids Research, Volume 45, Issue D1, January 2017, pages D219-D227.

[6] F. Meng, V.N. Uversky, L. Kurgan: "Comprehensive review of methods for prediction of intrinsic disorder and its molecular functions", Cellular and Molecular Life Sciences volume 74, pages 3069-3090(2017). 